\section{Integrating small Unmanned Air- Craft Systems (sUAS) into Meteorology and Atmospheric Science: Challenges and Opportunities for GIScience}

Gl_Forum 2017, Issue 2 Page: 189 - 199

Full Paper

Corresponding Author: amy.e.frazier@okstate.edu DOI: 10.1553/giscience2017_02_s189

\author{
Amy E. Frazier', Adam J. Mathews' ${ }^{1}$, Benjamin L. Hemingway', Christopher Crick², \\ Elinor Martin ${ }^{3}$ and Suzanne W. Smith 4 \\ 'Department of Geography, Center for Applications of Remote Sensing, Oklahoma State \\ University, USA \\ 2Department of Computer Science, Oklahoma State University, USA \\ ${ }^{3}$ School of Meteorology, University of Oklahoma, USA \\ ${ }^{4}$ Department of Mechanical Engineering, University of Kentucky, USA
}

\begin{abstract}
Small unmanned aircraft (or aerial) systems (sUAS) are poised to revolutionize geospatial data collection for meteorology and atmospheric science, particularly within the lowest portion of the atmosphere, referred to as the atmospheric boundary layer (ABL), where conventional data collection techniques such as satellites, balloons and ground-based weather towers do not provide adequate coverage for monitoring certain types of weather events. The prospects of these autonomous mobile sensing platforms for geospatial data collection in the atmospheric realm are immense, but there has been limited discourse on the broader GIScience-related research challenges and opportunities surrounding data capture and analysis for this field beyond traditional imagery applications. Here, we address the integration of sUAS into meteorology and atmospheric science applications, including severe weather monitoring, from the perspective of the myriad GIScience research challenges and opportunities that exist for collecting and processing alternative types of non-image geospatial data from sUAS. Specifically, we highlight opportunities surrounding data capture and processing, data representations, and societal implications stemming from these applications.
\end{abstract}

\title{
Keywords:
}

unmanned aerial vehicle (UAV), unmanned aircraft system (UAS), drones, meteorology, atmospheric physics, weather

\section{Introduction}

The atmospheric boundary layer (ABL), which is the portion of the atmosphere in direct contact with the surface of the Earth, plays a critical role in the formation of weather events. 
Approximately $1 \mathrm{~km}$ thick, the ABL is difficult to monitor using traditional tools such as weather balloons, ground-based radar, and networks of ground weather stations (i.e., mesonets), because these methods are often not able to sample the full extent of the ABL. Similarly, satellite-based imagery and radar do not offer adequate spatial and/or temporal resolutions for monitoring the small-scale changes in the ABL, which can occur on timescales as short as 20 minutes (Fujita, 1962). Small, unmanned aircraft systems (sUAS; weighing $<55 \mathrm{lbs}$ or $22.7 \mathrm{~kg}$ ) have the potential to be used as a diagnostic tool for atmospheric science and operational meteorology within the ABL, but there are many GIScience-related technical, conceptual, and societal challenges that need to be addressed before sUAS can be used to their full capacity in this realm. While sUAS have been used for many years in terrestrial remote sensing and have been successfully integrated into imagery data collection, processing, and analysis workflows (see Colomina \& Molina, 2014 for a review), there has been limited discourse on the broader research challenges and opportunities for integrating alternative, non-imagery data streams such as atmospheric measurements of temperature, humidity, and wind speeds into GIS analyses.

The aim of this article is to present a conceptual overview of some of the GIScience-related challenges and opportunities of integrating sUAS into meteorology and atmospheric science. While geospatial applications of sUAS have become practically synonymous with aerial photography surveys during recent years, there remain many theoretical, methodological, and ethical challenges for collecting and processing non-imagery datasets derived from sUAS. We discuss several specific areas of GIScience, including data capture and processing, data representation, and societal implications, where we see potential for advancements to be made surrounding the use of sUAS. We focus our discussion specifically on meteorology and atmospheric science, which have been under-examined in certain aspects of GIScience (Mark, Smith, Egenhofer \& Hirtle, 2005). It should be noted that the intent of this discussion is to stimulate increased sUAS-related discourse and investigation within the GIScience community, not necessarily to provide solutions to these challenges and opportunities.

\section{Data capture, storage, and processing}

Data acquisition and processing workflows for imagery collected from sUAS are discussed widely in the literature (e.g., structure from motion [SfM] algorithms, etc.), but other types of data acquisition from sUAS have not received comparable attention in the GIScience literature. In the atmospheric sciences, sUAS are being outfitted with sensors that measure temperature, pressure, humidity, and wind speed (Frew, Elston, Argrow, Houston \& Rasmussen, 2012; Hemingway, Frazier, Elbing, \& Jacob, 2017), as well as dropsondes (Avery \& Jacob, 2015), which are expendable weather reconnaissance devices that measure atmospheric conditions as they fall to earth (Figure 1). Scientists are specifically interested in using sUAS to capture vertical profiles of atmospheric conditions (Cassano, 2014), but challenges arise during data collection because researchers are only in the initial stages of 
establishing the optimal spatial sampling scales to adequately capture the phenomena under investigation (Hemingway et al., 2017). Determining appropriate data collection and sampling scales for spatial statistical analyses has historically been on GIScience research agendas (McMaster \& Usery, 2005; Goodchild, 2010), and considerable advancements have been made in terms of terrestrial sampling scales. However, with the limited attention given to meteorological phenomena in certain GIScience foci (Mark et al., 2005), there are opportunities for research, particularly related to sampling scales, that until now were not possible using available technologies. For example, a recent study utilized a common geostatistical technique, variogram modelling, to identify the optimal vertical scales from which to sample certain atmospheric phenomena (i.e., temperature and relative humidity) via sUAS flying profiles (Hemingway et al., 2017). Broadly, the authors found that vertical sampling scales of approximately 1-3 $\mathrm{m}$ were sufficient for capturing variation in temperature and relative humidity across their study area. However, they only investigated spatial autocorrelation in a single (i.e., vertical) direction for a single geographic area, and further investigations are needed to extend these findings to two, and eventually three (i.e., $x$, $y, z)$, dimensions across a wide range of environmental conditions.

a) Atmospheric sensor mounted on UAS

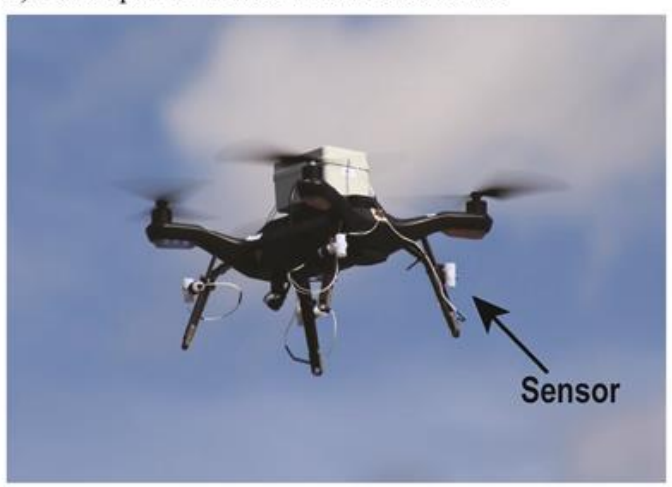

b) Dropsonde deployed from UAS

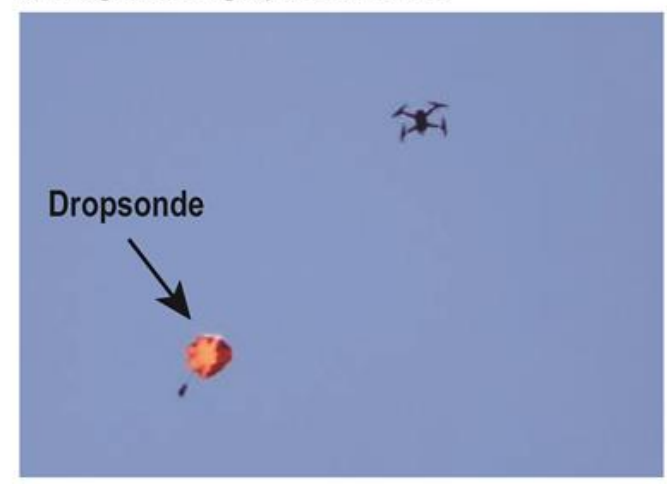

Figure 1: Atmospheric sensing from unmanned aerial systems (UAS). Photo credits: Jamey Jacob and CLOUD-MAP

Previously, the high cost of covering large areas through ground-based networks hindered widespread implementation of sensor networks (Wang, Zhang, \& Wang, 2006), but with advances being made in the development of low-cost, miniaturized sensing devices (Nittel, 2009), sUAS are providing a more dynamic and flexible option for implementing large sensor networks. Unlike ground-based weather stations in which towers, typically about $10 \mathrm{~m}$ high, are erected in a fixed geographic location and instrumented with various atmospheric sensors (McPherson et al., 2007), sUAS can carry the same suite of sensors with greater flexibility for covering space (Figure 2). sUAS also have greater flexibility in the spatial and temporal resolutions of the data captured compared to satellites, in which spatial resolution is often fixed before sensor deployment and cannot be changed after the satellite is launched. 
Frazier et al

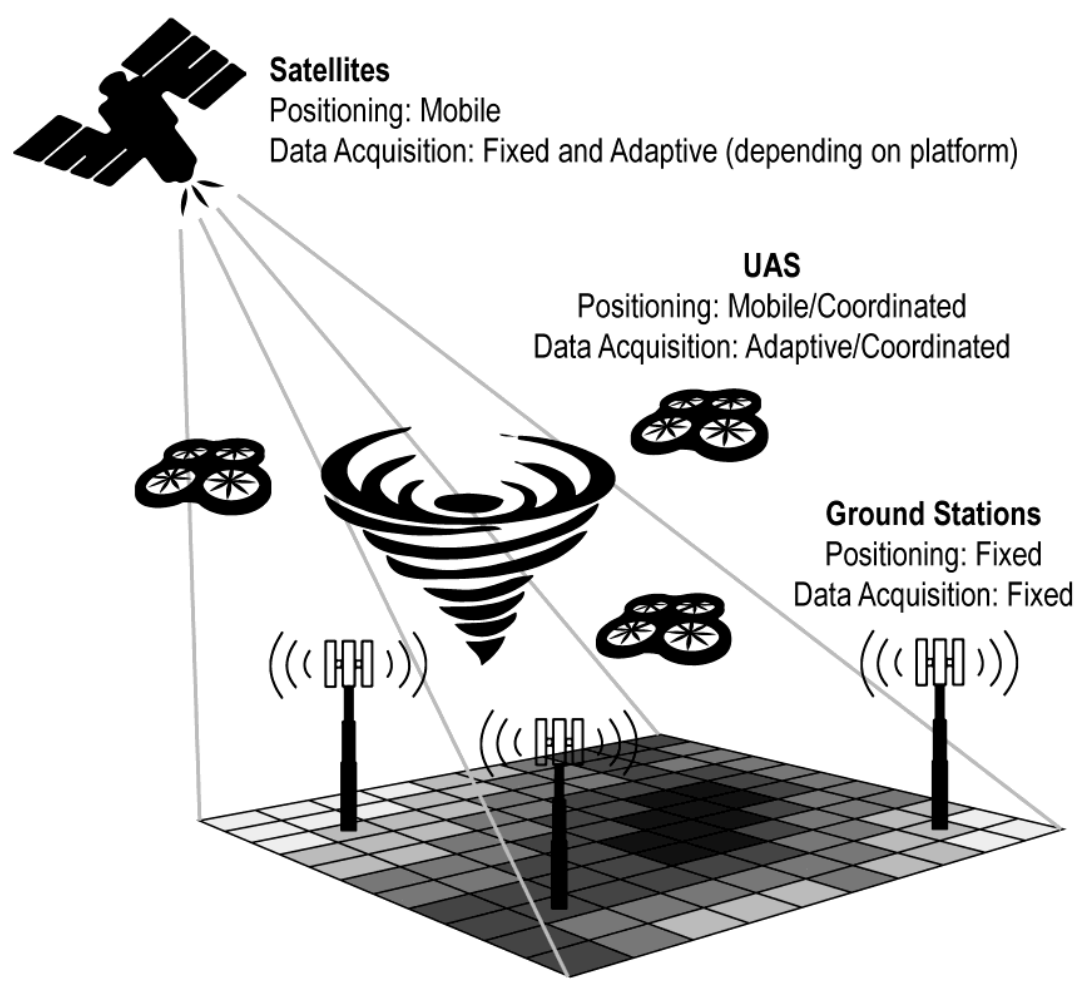

Figure 2: sUAS flying in coordinated networks can provide flexible positioning and data acquisition compared to satellites and ground stations

Another benefit of situating sensors onboard sUAS is the potential for adaptive sampling schemes via coordinated, self-organizing fleets of sUAS. Key to the implementation of these adaptive sUAS sensor networks is the development of algorithms for coordinating, controlling, and systematizing a distributed network of airborne sensors. Although development is ongoing, we anticipate that multiple sUAS flying in a mobile network will soon be able to communicate with each other and ground stations in order to autonomously coordinate collection of optimally distributed spatial datasets. This type of (semi)autonomous decision-making regarding sampling locations will permit adaptive sampling schemes, both atmospheric and terrestrial, not previously possible through fixed, groundbased networks. For atmospheric sampling in particular, sUAS will be able to communicate the information collected from onboard sensors (Figure 1a), such as temperature, humidity, and wind speed, to associated sUAS flying nearby; based on the incoming information, the associated sUAS will be able to alter their flight paths autonomously to identify the scale of the phenomenon of interest. This type of coordinated intelligence will ultimately be able to detect atmospheric phenomena, such as a storm supercell or pollution plume, much more rapidly than current systems allow. 
However, several GIScience-related challenges need to be overcome before we see fleets of data-collecting sUAS in the sky (Namuduri, Wan, \& Gomathisankaran, 2013). Current technology allows sUAS to fly in coordinated networks (e.g., sense and avoid, situational awareness), but the sensor, control, and antenna technologies that will enable multiple sUAS to make coordinated measurements are still being refined (Mullen, Bailey, \& Hoagg, 2016). Furthermore, collecting these data requires algorithms that can handle large variances in communication rates, link reliability, mesh network connectivity, and bandwidth (Crick \& Pfeffer, 2003), difficulties that are common when flying UAS, typically in rural areas. GIScience has long dealt with big data issues, particularly related to CyberGIS (Wang, 2010), but difficulties associated with storage, sequencing, integration, and communication are magnified when data are produced by mobile platforms with infrequent, unreliable bandwidth or power-limited message transfer. Data can be stored onboard all but the smallest, most limited platforms, as technical issues of weight, volume, and power consumption have largely been solved. However, data stored onboard are inaccessible to real-time applications, thus inhibiting cutting-edge, space-time GIScience applications. In other situations where data are transmitted over a radio connection, platforms may have to prioritize reporting, applying information-gain filters or novelty detection to prioritize data for communication, which again poses challenges for real-time geospatial analyses. Assuming these technological limitations can be overcome, opportunities exist in GIScience to investigate adaptive sampling schemes across space and time, and specifically within CyberGIS, to contribute to advances in distributed, mobile computing onboard sUAS.

\section{Geographic representation}

Regardless of whether researchers are flying a single sUAS on an isolated mission or coordinating a distributed, mobile network consisting of a fleet of sUAS collecting atmospheric data in tandem with each other across larger geographic regions, the data being collected for atmospheric and meteorological purposes typically consist of geographic (x, y, z) locations attributed with values for the particular phenomenon being sampled (temperature, humidity, wind speed, etc.). The output from these data-capture missions is a cloud of vector points, which is not unlike a point cloud produced by lidar returns, or the popular structure from motion (SfM) approach for creating 3D models of digital terrain and surfaces via aerial photographs (Jensen \& Mathews, 2016). Unlike discrete return lidar products or SfM point clouds that are often used to model objects on the surface of the earth (e.g., trees, buildings), the vector datasets produced from atmospheric investigations often represent continuous phenomena that vary in the $\mathrm{x}, \mathrm{y}$, and $\mathrm{z}$ dimensions. While some types of lidar returns, such as those representing elevation, are often converted into $2 \mathrm{D}$ rasters representing a continuous surface of elevation changes, the volumetric nature of atmospheric sampling limits the value of these types of rasters for representing atmospheric phenomena. Thus, neither 2D polygon objects nor 2D rasters are optimal for representing atmospheric datasets captured from sUAS. 
Voxels (i.e., volume elements) are currently the primary data model used to represent 3D objects in GIS, where each voxel represents a 3-dimensional cell in a tessellation, typically a regular tessellation, and the size of each voxel (i.e. the area it covers) is predetermined by the data model. Voxels can be interpolated across space from $x, y, z$ points or generated using a 'flood-filling' algorithm (Lieberwirth, 2008), but regardless of how they are created, they face similar challenges to those of $2 \mathrm{D}$ rasters, because resolution is predetermined, and pixels are often arbitrarily defined spatial units that do not always represent meaningful geographic units (Hay \& Castilla, 2008).

Voxels have seen limited use in the geographical domain, and the lack of adoption may be because their positional accuracy can be low if resolution is low, and because voxels cannot represent topological relationships, which form the backbone of spatial analysis (Gong \& Xia, 1999). Recognizing the limitations of 3D modelling in GIS, more than a decade ago Yuan, Mark, Egenhofer and Peuquet (2005) identified the need to improve geographic representations in order to better model volumetric (3D) and dynamic phenomena within GIScience, but progress has been slow. Most software packages still do not treat 3D or dynamic phenomena adequately, which is problematic for representing atmospheric data captured by sUAS.

Nearly two decades ago, Gong and Xia (1999) introduced an alternative data model for 3D representation, derived from integrating vector, raster, and object-oriented data models. Widespread adoption of their data model did not occur, and developing similar creative alternatives in which different types of spatial objects can be represented more completely and accurately than through voxels alone is an overdue research need in GIScience. In particular, prisms are a promising solution for modelling atmospheric data, since they can coincide with the square spacing of a $2 \mathrm{D}$ raster in an $\mathrm{x}, \mathrm{y}$ plane but model the $\mathrm{z}$ direction (height) with more precision. Recently, data model development activity has increased within open source platforms such as GRASS GIS (Neteler, Bowman, Landa, \& Metz, 2012), but sUAS may provide the needed technological stimulus to promote better modelling of 3D and dynamic phenomena within GIScience (see Nittel et al., 2015).

Dynamic and continuous representations are another area where the current GIS data models are sub-optimal for the types of atmospheric data which sUAS are anticipated to generate in the near future. As stated previously, state-of-the-art research in robotics and antenna design is expected to enable multiple sUAS to organize themselves during flight and collect coordinated, continuous measurements of environmental parameters, which, for atmospheric monitoring, would take the form of either linear or polygonal gradients of temperature or humidity. These types of gradient measurements are especially useful in severe weather monitoring to ensure platforms remain out of the direct path of the storm while still collecting valuable information in and around the storm (Figure 3). Twodimensional, discrete vector data models (i.e., arcs or lines) are unable to model and represent continuous, dynamic data in GIS. Single-row raster or voxel datasets (2D or 3D) are similarly inappropriate, since precision depends on the resolution of the pixels/voxels. 
Thus, new data models are needed that can appropriately abstract these representations of the environment.
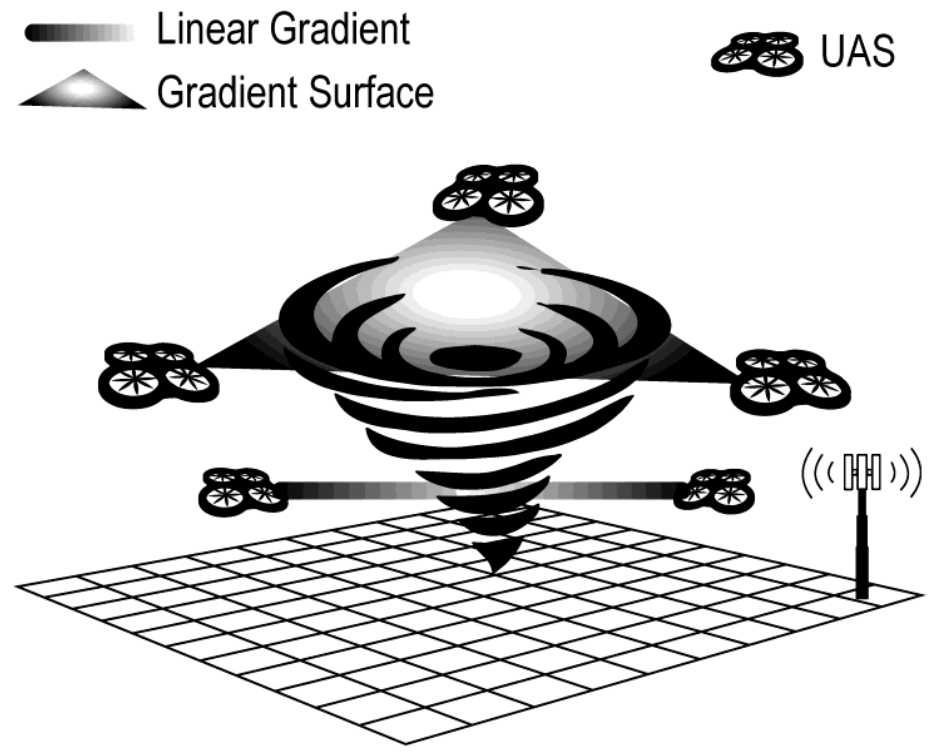

Figure 3: Hypothetical examples of continuous spatial data models obtained via coordinated networks of unmanned aerial systems (UAS) collecting data in tandem.

Lastly, acoustic and infrasonic waves are data types that have not been modelled in GIS but are likely to see increased collection via sUAS for applications related to atmospheric physics. Many types of natural and anthropogenic phenomena emit infrasound (e.g., avalanches, earthquakes, tornadoes, meteors, explosions, supersonic aircraft; Bedard, 1998), and cuttingedge research is investigating whether infrasound can be used to detect certain severe weather or seismic events (e.g., tornadoes or earthquakes) before they occur (Elbing \& Gaeta, 2016). This work will involve detecting and mapping the presence and degradation of infrasound waves via the integration of a ground sensor network with infrasonic sensors placed onboard sUAS platforms. However, the capabilities of mapping these data are limited, for several reasons. First, sound waves exhibit different characteristics from light waves (e.g., echoes), which may limit the usefulness of vector or raster data models for representing the distance and direction to a particular source of infrasound. Second, infrasound can travel long distances without dissipating, which makes it difficult to assign a spatial location to the source based solely on the measurement from a single sensor, and multiple arrays are often needed to triangulate the signal. However, if we can capture these signals and represent acoustic and infrasonic phenomena in GIS, it may be possible to integrate this information into weather models and warning systems. 


\section{$4 \quad$ Societal implications}

GIScience has been addressing questions of the privacy and confidentiality of geospatial data and surveillance using geotechnologies for decades (Elmes et al., 2005). Given the many ethical debates surrounding military drones, the use of sUAS for geospatial data collection has motivated renewed discussions of privacy issues with regard to the collection, use, and analysis of geospatial data. In a qualitative study about the privacy implications of UAS, Wang, Xia, Yao, and Huang (2016) found that territoriality (public vs. private spaces) played a key role in shaping participants' privacy concerns in relation to UAS. Unmanned systems flying within 'private spaces' (defined loosely by ownership of the space, sensitivity of the space, and activities performed within the space) were considered by respondents to be a greater invasion of privacy than those flying in 'pubic spaces'. However, if sUAS are to be integrated successfully into atmospheric research and, in particular, into severe weather monitoring, flights cannot be confined to public spaces if they are to capture valuable data. Thus, public awareness and acceptance of sUAS, including the public safety benefits they may provide and their usefulness in protecting assets, are critical for progressing towards routine flight operations (DeBusk, 2010).

A recent study by PytlikZillig, Duncan, Elbaum, and Detweiler (in review) gathered public perceptions from more than 800 respondents regarding the use of sUAS in weather-related monitoring. The authors found that the purpose of a mission impacts people's willingness to 'allow' or 'forgive' certain uses, with weather monitoring scoring high in terms of acceptability. These findings echo those of Miethe, Lieberman, Sakiyama, and Troshynski (2014), who report that $87 \%$ of the public support 'climatic/geological mapping' from sUAS. Further, PytlikZillig et al. (in review) found that certain trust-brokering processes, in which trust between the agencies performing the monitoring and the people that may be affected is strengthened, may, in certain situations that benefit society, have the potential to alleviate some of the negative perceptions surrounding the use of sUAS.

Lastly, societal challenges of incorporating sUAS into atmospheric science applications extend to the citizen science realm, where citizens operating their own sUAS system can contribute data in support of science missions. Citizen science fits within the subfield of Volunteered Geographic Information, or VGI (Goodchild, 2007), and an emerging research theme within these sub-fields is data quality and uncertainty (Haklay, 2013). While a substantial portion of the sUAS market is driven by civilian use of sUAS for photography, video, surveying, and mapping, there are relatively few citizen science and participatory mapping projects engaging with sUAS (Mathews \& Frazier, 2017). A probable reason is that these sorts of citizen science activities may pose problems of data quality and uncertainty. Specifically, uncertainties can be introduced at three stages by citizen scientists using sUAS for atmospheric science missions: (1) during the interpretation of atmospheric phenomena (e.g., identifying whether or not an atmospheric or severe weather event is occurring); (2) during the measurement and representation of atmospheric phenomena (e.g., ensuring onboard sensors are properly calibrated and collecting valid data); (3) during the post- 
processing and analysis of the data representing these phenomena (e.g., preventing mistakes during data download, transfer, upload, etc.). While some of the uncertainties experienced by sUAS operators are similar to those encountered when using other geospatial technologies (e.g. GPS), others, such as identifying atmospheric phenomena and calibrating sensors, may require specialized training to expand sUAS technology into VGI and citizen science projects.

\section{Conclusions}

Small, unmanned aircraft systems (sUAS) are a low-cost, flexible option for geospatial data collection that are rapidly being integrated into meteorology and atmospheric science, particularly for sensing the lowest portion of the atmosphere, the atmospheric boundary layer (ABL), where conventional techniques such as satellites, balloons, and ground-based weather towers do not provide adequate coverage. Their growing use in these domains presents both challenges and opportunities for GIScience, because the data being collected are typically not imagery, which is where most application-related sUAS advancements have been made in the GIS realm. In this paper, we have addressed some of the challenges associated with collecting and processing these alternative geospatial datasets acquired from sUAS and have also outlined some of the emerging opportunities for theoretical and methodological advances related to this technology, specifically focusing on non-image data capture for meteorology and atmospheric science. The areas of GIScience where we found a particular need for additional research include: (1) spatial sampling scales for capturing atmospheric phenomena via sUAS in both the vertical and horizontal dimensions; (2) CyberGIS challenges of providing real-time solutions for data storage, sequencing, system integration, and communication amongst a sensor network of sUAS; (3) societal implications, including privacy issues, trust, and citizen science applications. The aim of this study is to stimulate further sUAS-related discussion and investigations as this rapidly emerging technology is being adopted into new fields.

\section{Acknowledgements}

This work was supported by the United States National Science Foundation (NSF) Established Program to Stimulate Competitive Research (EPSCoR) under Grant [IIA1539070] 'RII Track-2 FEC: Unmanned Aircraft Systems for Atmospheric Physics'. We would like to thank Drs Jamey Jacob and Brian Elbing for thoughtful discussions that improved the manuscript greatly. 


\section{References}

Avery, A. \& Jacob, J. (2015). Design of a severe storm sesearch UAS. In proceedings of 53rd AIAA Aerospace Sciences Meeting, AIAA SciTech Forum, Kissimmee, FL, USA (p. 1905). https://doi.org/10.2514/6.2015-1905

Bedard Jr., A. J. (1998). Infrasonic and near infrasonic atmospheric sounding and imaging. Proceedings of Progress in Electromagnetics Research Symposium, 13-17. Retrieved from http://www.esrl.noaa.gov/psd/programs/infrasound/infrasonic.html

Cassano, J. J. (2014). Observations of atmospheric boundary layer temperature profiles with a small unmanned aerial vehicle. Antarctic Science, 26, 205-213

Colomina, I., \& Molina, P. (2014). Unmanned aerial systems for photogrammetry and remote sensing: A review. ISPRS Journal of Photogrammetry and Remote Sensing, 92, 79-97. DOI: 10.1016/j.isprsjprs.2014.02.013

Crick, C., \& Pfeffer, A. (2003). Loopy belief propagation as a basis for communication in sensor networks. Proceedings of the 19th Conference on Uncertainty in AI, 159-166. Retrieved from http://www.acm.org

DeBusk, W. M. (2010). Unmanned aerial vehicle systems for disaster relief: Tornado alley. Paper presented at the AIAA Infotech@ Aerospace Conference, Atlanta, GA

Elbing, B. R., \& Gaeta, R. J. (2016). Integration of Infrasonic Sensing with UAS. In 8th AIAA Atmospheric and Space Environments Conference (p. 3581) AIAA AVIATION Forum, (AIAA 2016-3581) http://dx.doi.org/10.2514/6.2016-3581

Elmes, G. A., Epstein, E., McMaster, R. B., Niemann, B. J., Poore, B., Sheppard, E., \& Tulloch, D. (2005). GIS and Society: Interrelation, integration, and transformation. In R. B. McMaster. \& L. Usery (Eds.), A Research Agenda for Geographic Information Science, 287-312. Boca Raton, FL: CRC Press

Frew, E. W., Elston, J., Argrow, B., Houston, A., \& Rasmussen, E. (2012). Sampling severe local storms and related phenomena: Using unmanned aircraft systems. IEEE Robotics and Automation Magazine, 19, 85-95.

Fujita, T. T. (1962). A Review of Researches on Analytical Mesometeorology; Mesometeorology Project: Department of the Geophysical Sciences, University of Chicago, Chicago, IL, USA

Gong, J. \& Xia, Z. (1999). An integrated data model in three dimensional GIS. Geo-spatial Information Science, 2, 1-8. DOI: 10.1007/BF02826710

Goodchild, M. F. (2007). Citizens as sensors: the world of volunteered geography. GeoJournal, 69(4), 211-221

Goodchild, M. F. (2010). Twenty years of progress: GIScience in 2010. Journal of Spatial Information Science, 1, 3-20

Haklay, M. (2013). Citizen science and volunteered geographic information: Overview and typology of participation. In Sui, D., Elwood, S., \& Goodchild, M. (Eds.), Crowdsourcing Geographic Knowledge. Dordrecht, Netherlands: Springer

Hay, G. J. \& Castilla, G. (2008). Geographic object-based image analysis (GEOBIA). A new name for a new discipline. In Blaschke, T., Lang, S., \& Hays, G. J. (Eds.), Object-Based Image Analysis. Berlin: Springer

Hemingway, B. L., Frazier, A. E., Elbing, B. R., \& Jacob, J.D. (2017). Vertical Sampling Scales for Atmospheric Boundary Layer Measurements from Small Unmanned Aircraft Systems (sUAS). Atmosphere, 8(9), 176-194

Jensen, J. L. \& Mathews, A. J. (2016). Assessment of Image-Based Point Cloud Products to Generate a Bare Earth Surface and Estimate Canopy Heights in a Woodland Ecosystem. Remote Sensing, 8, 50. DOI:10.3390/rs8010050 
Lieberwirth, U. (2008). Voxel-based 3D GIS: modelling and analysis of archaeological stratigraphy. In Frischer, B. \& Dakouri-Hild, A. (Eds.), Beyond Illustration: 2D and 3D Digital Technologies as Tools for Discovery in Archaeology. Oxford: Archaeopress

Mark, D. M., Smith, B., Egenhofer, M., \& Hirtle, S. (2005). Ontological foundations for geographic information science. In McMaster, R. B. \& Usery, E. L. (Eds.), A Research Agenda for Geographic Information Science. New York, NY: CRC Press

Mathews, A. J. \& Frazier, A. E. (2017). Unmanned Aerial Systems. In Wilson, J.P. (Ed.), The Geographic Information Science \& Technology Body of Knowledge (2nd Quarter 2017 Edition). Retried from: http://gistbok.ucgis.org/bok-topics/unmanned-aerial-systems-uas

McMaster, R. B. \& Usery, E. L. (2005). A Research Agenda for Geographic Information Science. New York, NY: CRC Press

McPherson, R. A., Fiebrich, C. A., Crawford, K. C., Kilby, J. R., Grimsley, D. L., Martinez, J. E., Basara, J. B., Illston, B. G., Morris, D. A., \& Kloesel, K. A. (2007). Statewide monitoring of the mesoscale environment: A technical update on the Oklahoma Mesonet. Journal of Atmospheric and Oceanic Technology, 24, 301-321

Miethe, T. D., Lieberman, J. D., Sakiyama, M., \& Troshynski, E. I. (2014). Public Attitudes about Aerial Drone Activities: Results of a National Survey. UNLV Center for Crime and Justice Policy: Research in Brief. Retrieved from: https://www.unlv.edu/sites/default/files/page_files/27/PublicAttitudesAboutAerialDroneActivi ties.pdf [last accessed: 1 October 2017]

Mullen, J., Bailey, S. C. C., \& Hoagg, J. B. (2016). Filtered dynamic inversion for altitude control of fixed-wing unmanned air vehicles. Aerospace Science and Technology, 54, 241-252. DOI: 10.1016/j.ast.2016.04.013

Namuduri, K., Wan, Y., \& Gomathisankaran, M. (2013). Mobile ad hoc networks in the sky: State of the art, opportunities, and challenges. Proceedings of the second ACM MobiHoc workshop on Airborne networks and communications, 25-28

Neteler, M., Bowman, M. H., Landa, M., \& Metz, M. (2012). GRASS GIS: A multi-purpose open source GIS. Environmental Modelling \& Software, 31, 124-130. DOI: 10.1016/j.envsoft.2011.11.014

Nittel, S. (2009). A survey of geosensor networks: Advances in dynamic environmental monitoring. Sensors, 9, 5664-5678. DOI: 10.3390/s90705664

Nittel, S., Bodum, L., Clarke, K., Gould, M., Rapaso, P., Sharma, J., \& Vasardani, M. (2015, September). 'Emerging technological trends likely to affect GIScience in the next 20years'. Paper presented at the meeting of the International Early Career Scholars Summer Institutes in Geographic Information Vespucci Institute, Bar Harbor, ME

PytlikZillig, L. M., Duncan, B. A., Elbaum, S., \& Detweiler, C. (In Review) “A drone by any other name: Purposes and end-users of UAVs affect public support," IEEE Technology and Society Magazine

Wang, N., Zhang, N., \& Wang, M. (2006). Wireless sensors in agriculture and food industry-Recent development and future perspective. Computers and Electronics in Agriculture, 50(1), 1-14

Wang, S. (2010). A CyberGIS Framework for the Synthesis of cyberinfrastructure, GIS, and spatial analysis. Annals of the Association of American Geographers, 100(3), 535-557

Wang, Y., Xia, H., Yao, Y., \& Huang, Y. (2016). Flying eyes and hidden controllers: A qualitative study of people's privacy perceptions of civilian drones. U.S. Proceedings on Privacy Enhancing Technologies, 3, 172-19

Yuan, M., Mark, D. M., Egenhofer, M. J., \& Peuquet, D. J. (2005). Extensions to Geographic Representation. In McMaster, R. B. \& Usery, E. L. (Eds.), A Research Agenda for Geographic Information Science. New York, NY: CRC Press 